\title{
Assessing behavioral control across reinforcer solutions on a fixed-ratio schedule of reinforcement in rats
}

\author{
Joel E. Shillinglaw ${ }^{1}$, lan K. Everitt ${ }^{1}$, and Donita L. Robinson ${ }^{1,2,{ }^{*}}$ \\ ${ }^{1}$ Bowles Center for Alcohol Studies, University of North Carolina, Chapel Hill, NC, USA \\ ${ }^{2}$ Department of Psychiatry, University of North Carolina, Chapel Hill, NC, USA
}

\section{Abstract}

Instrumental behavior can shift from flexible, goal-directed actions to automatic, stimulusresponse actions. The satiety-specific devaluation test assesses behavioral flexibility by evaluating reward seeking after temporary devaluation of the reinforcer via satiety; a decrease in responding compared to control conditions indicates goal-directed behavior. We have observed variability in the outcome of this test that may be dependent on the reinforcer. Another test of habit, contingency degradation, involves changing the action-outcome association over the course of retraining and determines whether reward seeking is sensitive to changing contingencies. We hypothesized that the outcome of the contingency-degradation test would remain consistent across reinforcers, while the satiety-specific devaluation test may vary across reinforcers because it depends on the ability of the reinforcer to induce satiety. Therefore, we trained rats to selfadminister $1.5 \%$ sucrose, $10 \%$ sucrose, $10 \%$ ethanol, or $10 \mathrm{mM}$ monosodium glutamate (MSG) on a fixed-ratio (FR5) schedule that has been shown to promote long-term, goal-directed responding. Next, behavioral flexibility was evaluated in three satiety-specific devaluation tests over 6 weeks. Finally, we investigated reward seeking after contingency-degradation training. All groups displayed sensitivity to satiety-specific devaluation in the first test, indicating goal-directed behavior. While the $10 \%$ sucrose and ethanol groups remained goal-directed, the $1.5 \%$ sucrose and MSG groups exhibited habit-like behavior in later tests. Nevertheless, all groups displayed decreased responding in an extinction session after contingency-degradation training, indicating goal-directed behavior. These results demonstrate that tests of behavioral flexibility can yield dissimilar results in the same rats. Next, rats from the $1.5 \%$ sucrose group underwent the entire experiment again, now self-administering $10 \%$ sucrose. These rats showed pronounced goaldirected behavior in satiety-specific and contingency-degradation tests under $10 \%$ sucrose conditions, further suggesting that the reinforcer solution affected the outcome of the satietyspecific devaluation test. We conclude that reinforcer characteristics should be considered when investigating habit-like behavior in alcohol research.

\section{(C) 2014 Elsevier Inc. All rights reserved.}

*Corresponding author: Donita L. Robinson, Bowles Center for Alcohol Studies, CB \#7178, University of North Carolina, Chapel Hill, NC, 27599-7178, Telephone: +1 919966 9178, Fax: +1919966 5679, DLR@unc.edu.

Publisher's Disclaimer: This is a PDF file of an unedited manuscript that has been accepted for publication. As a service to our customers we are providing this early version of the manuscript. The manuscript will undergo copyediting, typesetting, and review of the resulting proof before it is published in its final citable form. Please note that during the production process errors may be discovered which could affect the content, and all legal disclaimers that apply to the journal pertain. 


\section{Keywords}

ethanol; monosodium glutamate; sucrose; maltodextrin; satiety; operant

\section{Introduction}

Flexible, outcome-driven behaviors may shift to more inflexible, environmentally driven habits with repeated performance. Such a transition in the locus of control is implicated in individuals with alcohol use disorder, whose drinking persists despite significant negative consequences. A behavior may be described as either goal- or habit-directed by its sensitivity to changes in expectation. Accordingly, these behaviors can be evaluated in animal models of reinforcement by manipulating an aspect of reward expectation, typically the subjective value of the outcome or the contingency between behavior and outcome (Dickinson, 1985; Dickinson, Wood, \& Smith, 2002; Yin \& Knowlton, 2006; Yin, Knowlton, \& Balleine, 2006). Since the rate of reinforcement in fixed or random ratio schedules is proportional to the rate of responding, these schedules provide immediate feedback and drive flexible, "goal-directed" behaviors. Conversely, in variable or random interval schedules, reinforcement is additionally dependent on the passage of time; as a result, the correlation between responding and reinforcement is weakly perceived and yields more inflexible, "habit-like" behaviors. Indeed, the degree of "goal" or "habit"-directedness is often determined by the change in responding under extinction conditions following manipulation of either the outcome value or the action-outcome contingency, with larger and smaller changes reflecting goal-directed and habit-like seeking, respectively.

The outcome value in instrumental conditioning may be either increased or decreased, but is typically decreased to test for goal-directed behavior. Conditioned taste aversion, a technique that pairs the reinforcer with a lithium chloride injection, can be an effective method of devaluation but often produces reductions in self-administration lasting days to weeks (Foy \& Foy, 2009; Nolan et al., 1997). In contrast, satiety-specific devaluation transiently decreases the value of a reinforcer by inducing satiety via free access. Because consumed foods are subsequently both less desired and consumed relative to other palatable foods when given free access (Rolls, Rolls, Rowe, \& Sweeney, 1981), satiety is to a large degree taste-specific. Reward-seeking can be tested after free access to the reinforcer (satiety) and compared to seeking after access to an alternative solution. In such reward devaluation tests, decreased responding after reinforcer devaluation relative to control conditions would indicate "goal-directed" behavior, while comparable responding across conditions would indicate "habitual" behavior.

Alternatively, contingency-degradation tests assess flexibility by evaluating reward-seeking behavior after degradation of the action-outcome contingency (Bradfield, Bertran-Gonzalez, Chieng, \& Balleine, 2013; Yin \& Knowlton, 2006). Degradation may be accomplished via uncoupling of reward delivery from responses, or even reversal of the contingency via omission, in which the withholding of a response results in reinforcement. The animal's behavior is assessed in extinction sessions before and after the contingency-degradation 
training. Again, decreases in seeking behavior after contingency degradation indicate "goaldirected" behavior, while insensitivity to the new training is considered "habit".

Despite initial differences in behavioral flexibility, both low and high contingency schedules can reflect habit-like behavior with repeated performance as animals become "overtrained" (Dickinson, 1985). Additionally, the properties of the reinforcer have been shown to influence the rate of habit formation, as ethanol reinforcement appears to promote habit formation as compared to non-drug reinforcers (Corbit, Nie, \& Janak, 2012; Dickinson, Wood, \& Smith, 2002; Mangieri, Cofresí, \& Gonzales, 2012) (but see Samson et al., 2004). However, low doses of ethanol may be insufficient to accelerate habit formation, as rats selfadministering $\sim 0.5 \mathrm{~g} / \mathrm{kg}$ ethanol daily retained goal-directed behavior on an FR5 reinforcement schedule over several weeks (Hay, Jennings, Zitzman, Hodge, \& Robinson, 2013). Moreover, in that study and unpublished observations from our laboratory, we found that rats self-administering ethanol or 10\% sucrose on an FR5 reinforcement schedule appeared more sensitive to satiety-specific devaluation (i.e., more goal-directed) than rats self-administering $1.5 \%$ sucrose on the same schedule, suggesting that reinforcer properties may contribute to the assessment of behavioral flexibility. Since satiety-specific devaluation as a test of habit is dependent upon the ability of the solution to induce satiety and since our reinforcing solutions differ across short-term satiety signals like caloric load, it follows that the outcome of the devaluation test may vary across reinforcer solutions. However, as contingency degradation depends only upon the experience of changing contingencies, it is plausible this method would be more consistent across reinforcers.

The goal of the present study was to determine the congruency between satiety-specific devaluation and contingency-degradation tests of behavioral flexibility in rats trained to selfadminister one of four solutions on an FR5-reinforcement schedule. The solutions were $1.5 \%$ sucrose (w/v), $10 \%$ sucrose (w/v), $10 \mathrm{mM}$ monosodium glutamate (MSG), and 10\% ethanol (w/v) faded with either sucrose or MSG. MSG was chosen as a non-sweet, alternative reinforcer (McCool \& Chappell, 2012; Shibata, Kameishi, Kondoh, \& Torii, 2009) that has been used as an ethanol-fading solution (McCool \& Chappell, 2012). We hypothesized that the outcome of the satiety-specific devaluation test would be influenced by the reinforcer, whereas contingency degradation would not.

\section{Materials and Methods}

\section{Animal subjects}

Adult, male Long-Evans rats $(n=83)$ were obtained from Harlan Laboratories (Frederick, $\mathrm{MD)}$ at a starting weight range of 250-275 grams. Rats were individually housed in a controlled vivarium (12-h light/dark schedule with lights on at 7:00 AM; $25^{\circ} \mathrm{C}$ ) and had food and water ad libitum except as noted below. Rats were given 5 days upon arrival to adapt to the vivarium before training began ( 5 days/week, Monday-Friday). All procedures were compliant with the NIH Guide for the Care and Use of Laboratory Animals and approved by the Institutional Animal Care and Use Committee of the University of North Carolina at Chapel Hill. 


\section{FR5 Self-Administration Training}

Self-administration training occurred in operant boxes within sound-attenuating chambers (Med Associates, St. Albans, VT). Each box contained two retractable levers on one wall, with a circular cue light located above each lever and two metal fluid cups in between the levers. A house light was located on the upper portion of the opposite wall, and an exhaust fan and white noise generator were on during training sessions. Each training session began with a 5-min waiting period, followed by illumination of the house light and, after $30 \mathrm{~s}$, extension of the levers. At each reinforced lever-press response, the activated lever's cue light turned on, the house light turned off, both levers were retracted, and $0.1 \mathrm{~mL}$ fluid was dispensed into the activated lever's cup. After $5 \mathrm{~s}$, the levers extended, the cue light extinguished, the house light resumed, and the rat was able to again press the lever for reinforcement. Cups were checked at the end of each session to verify that the rat consumed the reinforcer and any remaining volume was recorded.

Rats were trained to respond for either $1.5 \%$ sucrose (w/v; $1.5-\mathrm{SUC}), 10 \%$ sucrose (w/v; $10-$ SUC), $10 \mathrm{mM}$ MSG (10-MSG), or 10\% ethanol (w/v; 10-E); 10-E self-administration was accomplished either by a typical sucrose fade (S-10-E) (Hay et al., 2013; Samson, 1986) or by an analogous MSG fade (M-10-E) (McCool \& Chappell, 2012). The training schedules for the five resulting experimental groups are shown in Table 1. For the first 5 days of training, animals were water-deprived up to $23 \mathrm{~h}$ per day to facilitate learning of the operant task, with 1-h water access immediately following the operant session. Sessions 1 and 2 were run on an FR1 reinforcement schedule, with sessions 3-8 run on an FR3 schedule before moving to the FR5 schedule that was maintained thereafter.

During the initial three training sessions, responding on either lever resulted in a fluid reward and the session lasted up to $3 \mathrm{~h}$. On the fourth day of training, the rats' least preferred side was used as the rewarded lever and on subsequent days the active lever alternated daily; these sessions were limited to $30 \mathrm{~m}$. For the S-10-E and M-10-E groups, concentration of ethanol gradually increased while the concentration of sucrose or MSG concurrently decreased. The 10-MSG and 1.5-SUC groups followed the M-10-E and S-10-E groups' fading procedures through the first 20 sessions but without the inclusion of ethanol. Finally, rats in the 10-SUC group were maintained for the duration of the experiment at the $10 \% \mathrm{w} / \mathrm{v}$ concentration from session 4 onwards. To equate the number of fluid deliveries earned across groups, sessions were limited to 50 or 25 reinforcements as shown in Table 1 .

\section{Satiety-Specific Devaluation}

Beginning at week 5 of training, rats underwent a satiety-specific devaluation test each Friday for 6 weeks (control vs. test solution across groups at weeks 5-6, 7-8, and 9-10), resulting in three consecutive assessments of sensitivity to reward devaluation, as previously described (Fanelli, Klein, Reese, \& Robinson, 2013; Hay et al., 2013). For each test, rats were allowed $60 \mathrm{~min}$ free access to their training reinforcer solution or a control solution ( $2 \%$ maltodextrin, w/v) from a sipper tube attached to the home cage, with solution presentation order balanced within groups and alternating each week. The maltodextrin solution served as a control for access to a palatable fluid before the test session, but it did not necessarily control for caloric intake; $2 \%$ maltodextrin has a similar caloric load to $1.5 \%$ 
sucrose. The available volume was $30 \mathrm{~mL}$ of the reinforcing fluid or maltodextrin, with the exception of S-10-E and M-10-E rats, which were given $15 \mathrm{~mL}$ of each solution; this adjusted volume was based on free-access drinking volumes observed in a previous study (Hay et al., 2013). Next, rats were placed in operant chambers and underwent a 5-min operant session under extinction conditions in which responses on both levers were counted but had no consequences (no cues or fluid deliveries). Lever-press responses after reinforcer access were compared to responses after maltodextrin access to assess sensitivity to satietyinduced reward devaluation. Lever-press responses during extinction were considered reward seeking and fewer responses after reinforcer-satiety versus maltodextrin-satiety were interpreted as goal-directed behavior. Conversely, similar lever pressing after reinforcer or maltodextrin access indicated habit-like behavior maintained by the operant environment. Sipper tubes filled with water were presented to the rats in their home cage once per week on Tuesday, Wednesday, or Thursday to prevent the rats from associating the sipper tube with a subsequent extinction session.

\section{Contingency Degradation Training}

On week 11, rats received regular self-administration sessions except for Wednesday, when they underwent a 10-min extinction test to assess reward seeking ("pre-test"). In week 12, rats had three daily sessions of contingency-degradation training as previously described (Fanelli et al., 2013). In these 30-min sessions, lever-press responses were counted but had no consequences (no cues or fluid deliveries), while rewards and associated cues were delivered on an RT30 schedule (cues and fluid aliquots were delivered randomly on an average interval of $30 \mathrm{~s}$ ). On the following day, rats were tested in a final 10-min extinction session to assess post-training reward-seeking behavior.

\section{Retraining Rats on $10 \%$ Sucrose}

Upon completion of contingency-degradation training, four 1.5-SUC animals were retrained on $10 \%$ sucrose and repeated the entire experiment (acquisition, maintenance, satietyspecific devaluation tests, contingency-degradation test) to further investigate reinforcer effects on the two tests of flexible behavior. All conditions were identical to 10-SUC trained animals.

\section{Data Analysis}

Lever-press responding during acquisition and maintenance of self-administration was compared across groups with a Kruskal-Wallis one-way ANOVA on ranks, due to nonnormal distribution of the data. Lever-press responding in tests of reward devaluation and contingency degradation and fluid volumes consumed prior to devaluation tests were compared across groups with a two-way ANOVA, using a mixed design (group: betweensubject; session or pre-access fluid: within-subject). Analyses were conducted in SigmaPlot 11.0 (Systat Software, Inc., San Jose, CA) and post hoc contrasts were corrected for multiple comparisons (Bonferroni correction). 


\section{Results}

\section{Acquisition and Maintenance of Self-Administration}

Only rats that achieved 25 reinforcer deliveries at least 3 of every 5 days after session 20 were used in the study. There was variability among groups in the proportion of rats to meet this criterion, as shown in Table 2. While the majority of rats self-administered sucrose (either $1.5 \%$ or $10 \%$ ) to criterion, only one-third of the rats assigned to the MSG, S-10-E, or M-10-E groups achieved performance criterion. Final group $n$ 's were: 1.5-SUC, $n=8 ; 10-$ SUC, $n=6$; MSG, $n=7$; S-10-E, $n=7$; M-10-E, $n=6$. Due to the response criterion, the 25-reinforcement limit per session, and the strict experimental timeline, training history across groups was similar, such that subsequent differences in behavioral responding were not due to differences in reinforcement history. Specifically, the number of reinforcements prior to the first devaluation test did not vary by group $\left(\mathrm{H}_{4}=0.76, p=0.94 ; 1.5-\mathrm{SUC}\right.$ : $665 \pm$ 27; 10-SUC: $693 \pm 19$; MSG: $681 \pm 40$; S-10-E: $664 \pm 41$; M-10-E: $671 \pm 46$ ).

To ensure that the repeated devaluation tests did not affect maintained self-administration of reinforcers, we compared the cumulative reinforcements earned in the regular sessions during the 6 weeks of devaluation testing. The number of earned reinforcements in these 6 weeks did not vary by group $\left(\mathrm{H}_{4}=3.8, p=0.43 ; 1.5\right.$-SUC: $496 \pm 3$; 10-SUC: $499 \pm 0.5$; MSG: $495 \pm 3$; S-10-E: $492 \pm 5$; M-10-E: $500 \pm 0$ ). Thus, in rats that met performance criteria, acquisition and maintenance of self-administration behavior did not vary by reinforcer fluid.

As the S-10-E and M-10-E groups did not differ in self-administration acquisition or maintenance, we pooled them into a single 10-E group for the analyses of behavioral flexibility. The dose of alcohol achieved during self-administration sessions varied by rat weight. Specifically, before the first devaluation test, rats in the 10-E groups weighed $410 \pm$ $5 \mathrm{~g}$ and achieved $0.61 \pm 0.01 \mathrm{~g} / \mathrm{kg}$ ethanol, and after the final devaluation test, they weighed $494 \pm 9 \mathrm{~g}$ and achieved $0.51 \pm 0.01 \mathrm{~g} / \mathrm{kg}$ ethanol. A previous study showed that blood ethanol content in rats self-administering $0.5 \mathrm{~g} / \mathrm{kg}$ ethanol under identical FR5 conditions was $\sim 30 \mathrm{mg} / \mathrm{dL}$ (Hay et al., 2013).

\section{Satiety-Specific Devaluation}

We examined differences in behavioral flexibility by using the satiety-specific devaluation test across groups three times: at weeks $5-6,7-8$, and 9-10. Rats were allowed to drink to satiety in the $60 \mathrm{~min}$ prior to the extinction test. Volumes consumed during this time are shown in Table 3. During each test, the two-way ANOVA revealed significant interactions of pre-access fluid by group on the volume consumed (Test 1 : $\mathrm{F}_{3,30}=7.7, p<0.001$; Test 2: $\mathrm{F}_{3,30}=6.2, p<0.005$; Test $3: \mathrm{F}_{3,30}=10.1, p<0.001$ ); for post hoc comparisons, see Table 3. Overall, volumes of maltodextrin consumed were similar across groups and devaluation tests. In contrast, volumes of reinforcer fluid volumes often differed, with lower volumes of MSG and 10-E consumed as compared to 1.5-SUC or 10-SUC.

When considering reward seeking during extinction after pre-access to the reinforcer or maltodextrin solutions, all groups were goal-directed in the initial test (Fig. 1, top), with rats exhibiting $15-42 \%$ fewer presses after devaluation versus control conditions. A two-way 
ANOVA yielded a main effect of pre-access solution $\left(\mathrm{F}_{1,30}=15.7, p<0.001\right)$, but no significant effect of group or group by solution interaction. However, in the second test, rats in the 1.5-SUC and MSG groups no longer showed differential lever-press responding after reinforcer access versus maltodextrin access (Fig. 1, middle). Specifically, a two-way ANOVA on responses yielded a significant group by solution interaction $\left(\mathrm{F}_{3,30}=6.1, p<\right.$ 0.005), and Bonferroni-corrected post hoc comparisons showed that lever presses were significantly reduced after reinforcer access (indicating devaluation) only in the 10-E and 10-SUC groups $(p<0.05)$. This pattern continued in the third devaluation test (Fig. 1, bottom): only the 10-E and 10-SUC groups exhibited reduced lever-press responding after reinforcer access (group by solution interaction: $\mathrm{F}_{3,30}=5.1, p<0.01$; Bonferroni-corrected post hoc comparisons within 10-SUC and 10-E groups, $p<0.05$ ). Thus, the 10-SUC and 10E groups exhibited goal-directed behavior across the three satiety-specific devaluation tests, while behavior in the 1.5-SUC and MSG groups was initially goal-directed but changed to habit-like in the later tests.

As satiety is regulated by multiple factors including caloric density, we compared the caloric load from the fluids consumed during the pre-access period to provide further explanation of disparate press behavior after free access. The solutions differed greatly in their caloric load, as shown in Table 3. Analysis of calories consumed prior to each devaluation test yielded significant interactions of group by pre-access solution: test $1\left(\mathrm{~F}_{3,30}=57.3, p<0.001\right)$, test $2\left(\mathrm{~F}_{3,30}=37.4, p<0.001\right)$, and test $3\left(\mathrm{~F}_{3,30}=108.7, p<0.001\right)$; see Table 3 for significant post hoc contrasts. Overall, the caloric load of maltodextrin consumption was similar across all groups. In contrast, the greatest calories resulted from 10-SUC followed by 10-E, with fewer calories from 1.5-SUC or MSG.

\section{Contingency Degradation}

Following the final devaluation, rats underwent a second measure of behavioral flexibility in which lever-press responding during two 10-min operant sessions under extinction conditions separated by 3 days of contingency-degradation training was compared. One MSG rat did not undergo degradation training due to experimenter error, and a computer error resulted in the loss of data from one training day of one 10-E rat (the missing data automatically handled by Sigma Plot by a general linear model). During the 3 days of contingency-degradation training, all groups exhibited reduced lever-press responses (Fig. 2, top). A two-way RM ANOVA revealed a significant main effect of day $\left(\mathrm{F}_{2,57}=23.8, p<\right.$ 0.001 ), but no significant effect of group or group by day interaction. Specifically, responding on Day 1 was greater than on Days 2 or 3 when collapsed across groups (Bonferroni-corrected $t$ tests, $p<0.05$ ). The critical test of behavioral flexibility was to determine whether the contingency-degradation training decreased reward seeking in the post-training extinction session in comparison to the pre-training session. All groups displayed significantly reduced responding in extinction following degradation training (Fig. 2, bottom, main effect of pre/post session, $\mathrm{F}_{1,29}=38.6, p<0.001$ ), but there was no significant effect of group or group by session interaction. These results demonstrate that all groups displayed sensitivity to contingency-degradation training, indicative of goal-directed behavior. 


\section{Retraining Rats on $10 \%$ Sucrose}

In the satiety-specific devaluation test, rats trained to self-administer 10-SUC remained goal directed while those trained to self-administer 1.5-SUC showed habit-like behavior. To test the role of sucrose concentration on the outcome of this test, four rats in the 1.5-SUC group (a subset) repeated the entire experiment under 10-SUC conditions. We compared behavioral flexibility in the satiety-specific devaluation test in these four rats under 1.5-SUC (1st training) and 10-SUC (retraining) reinforcement (Fig. 3). In this subset, the pattern of behavior in the three assessments under 1.5-SUC reinforcement was consistent with the full 1.5-SUC group data shown in Figure 1, with goal-directed behavior in the first test but habitlike behavior on subsequent tests. However, when these rats were retrained to 10-SUC, they exhibited goal-directed behavior across the devaluation tests. Specifically, a two-way ANOVA of presses in the first satiety-specific devaluation test showed that rats pressed less after reinforcer access than control access (main effect of access solution, $\mathrm{F}_{1,6}=35.1, p<$ 0.001; no significant effect of sucrose concentration or interaction). Analysis of presses in the second test revealed no significant differences between the two groups. Finally, the analysis of lever presses in the third devaluation test yielded a significant interaction of preaccess solution by sucrose concentration $\left(\mathrm{F}_{1,6}=9.6, p<0.05\right)$. Post hoc comparisons revealed that rats retained goal-directed behavior when trained to self-administer 10-SUC (presses after maltodextrin vs. 10-SUC access, $p<0.05$ ) but not 1.5-SUC (presses after maltodextrin vs. 1.5-SUC access, $p>0.05$ ). Thus, the same rats exhibited goal-directed or habit-directed behavior on the third devaluation test, depending on the concentration of sucrose on which they were trained and tested.

We also assessed behavioral flexibility after contingency-degradation training in these rats. When comparing lever-press responding in brief extinction sessions before versus after 3 days of contingency-degradation training, a two-way ANOVA revealed a significant main effect of session $\left(\mathrm{F}_{1,6}=26.0, p<0.005\right)$ but no significant effects of sucrose concentration or interaction. In other words, rats exhibited goal-directed behavior via this test regardless of the concentration of sucrose on which they were trained and tested. Together, these data suggest that the results from the satiety-specific devaluation test, but not the contingencydegradation test, are reinforcer-specific under the current experimental conditions.

\section{Discussion}

There is growing interest in the scientific community regarding the role of habit formation in addiction and alcohol drinking (Yin, 2008). Recent studies have investigated the contribution of habit in animal models of alcohol self-administration in an effort to determine how habit processes may impact both persistence of drinking and treatment efficacy (Corbit et al., 2012; Dickinson et al., 2002; Fanelli et al., 2013; Hay et al., 2013; Mangieri, Cofresí, \& Gonzales, 2012; Nam et al., 2013; Samson et al., 2004). However, there are multiple approaches to evaluate "habit" in animal models and their utility in alcohol studies has not been systematically evaluated. In the present study, we compared behavioral flexibility by two different methods - satiety-specific devaluation and contingency-degradation training - in rats with equivalent self-administration experience for either 1.5-SUC, 10-SUC, MSG, or 10-E. The major finding is that the satiety-specific 
devaluation and the contingency-degradation tests yielded conflicting results in some groups (1.5-SUC, MSG) but not others (10-SUC, 10-E). These results have implications for experimental design and interpretation of studies of habitual alcohol drinking and seeking in animals and suggest that a full evaluation of behavioral flexibility in a particular model may require multiple approaches.

Various tests are used to assess behavioral flexibility in rodent models, and most involve either reward devaluation or contingency degradation. The satiety-specific devaluation test devalues the reward by allowing the animal to consume the reward to satiety; this is an advantage over other tests of habit, as the devaluation is temporary. Specifically, it allows behavioral flexibility to be assessed at some point in training, and then continued selfadministration (Corbit et al., 2012) or extinction/reinstatement studies can follow (Hay et al., 2013). However, a potential problem of using the satiety-specific devaluation test with alcohol studies is that animals may become intoxicated after free access to alcohol, such that behavior may only appear to be goal-directed due to motor effects of intoxication. Thus, it is prudent to confirm the results of the satiety-specific devaluation test with those of a second test of habit in alcohol-drinking animals. The present study employed a reinforcement schedule (FR5, daily alternating lever) that has been shown to be habit-resistant (Fanelli et al., 2013; Hay et al., 2013), and all tests of habit - the three devaluation tests and the contingency-degradation training test - indicated that 10-E rats and 10-SUC rats were goaldirected. In contrast, rats in the 1.5-SUC and MSG groups exhibited goal-directed behavior only in the first devaluation test and the contingency-degradation test. A limitation of the present study is that the devaluation tests necessarily preceded the contingency-degradation tests, and the order was not reversed, although intuitively the new learning promoted by contingency-degradation training (Bradfield et al., 2013) seems more likely to interfere with subsequent self-administration than would satiety-specific devaluation testing. Of importance to alcohol studies is the difference in the 1.5-SUC and 10-SUC groups, as sucrose reinforcement is typically used as a non-drug control for alcohol self-administration studies. While lower concentrations of sucrose better match aspects of alcohol selfadministration, such as response rate (Besheer, Fisher, Lindsay, \& Cannady, 2013; Besheer et al., 2010; Corbit et al., 2012; Czachowski \& Delory, 2009), the present findings suggest that higher concentrations of sucrose better match the behavioral flexibility assessed after satiety-specific devaluation. One might next consider whether these results imply that the reinforcing value of stimuli (for which 1.5-SUC is a good match for 10-E) is "uncoupled" from inflexible responding for the same stimuli (for which 1.5-SUC is not a good match for 10-E). While this may be the case, we find that the present data do not suggest that they are necessarily uncoupled, as the contingency-degradation test yielded goal-directed behavior for all rats. Rather, we find that the more parsimonious explanation is that caloric content affects satiety and, thus, the specific satiety-devaluation test, as discussed below.

It is possible that, despite goal-directed behavior in the contingency-degradation test, rats self-administering 1.5-SUC and MSG developed habitual reward-seeking behavior, as indicated by insensitivity to reward devaluation exhibited in the second and third devaluation tests. Indeed, one factor in the shift from goal-directed to habitual reward seeking behavior is reinforcement history, and extended training can produce habitual responding via overtraining. We tested this possibility by retraining four rats in the 1.5 -SUC 
group to self-administer 10-SUC: these retrained rats would be biased toward habit-directed responding if affected by the total amount of prior self-administration training. Instead, these rats showed pronounced goal-directed behavior in satiety-specific and contingencydegradation tests under 10-SUC conditions. One caveat is that the change in behavior after 10-SUC devaluation was significant (and, thus, goal-directed) in the first and third tests, but not the second devaluation test; this inconsistency is likely due to the low number of rats in this part of the study. A second caveat is that we did not have a group of rats that continued on 1.5-SUC for a comparable amount of time. Such a control would allow for examination of potential reward contrast effects (Flaherty, 1982) or other factors that may have contributed to the outcome of the retraining to 10-SUC. For example, rats may have learned that when the reinforcer is presented in the home cage before a session, it will later receive an extinction session and, thus, learn not to press. Arguing against this possibility, one would expect such learning to also occur for exposure to maltodextrin in the home cage before a session, but rats pressed robustly after that exposure. As a proof-of-principle experiment, the retraining study was sufficient to demonstrate that behavioral flexibility in the same rats differed depending on the reinforcer solution. Thus, we conclude that the outcome of the satiety-specific devaluation test, especially after the first test, can vary based on the reinforcement solution.

We posit that in the present study all groups were goal-directed, as indicated by the initial satiety-specific devaluation test and the contingency-degradation tests. Why, then, did 1.5SUC and MSG rats show goal-directed behavior only in the first satiety-specific devaluation tests? Several factors contribute to satiation and satiety, including the orosensory perception of the food, its energy density, and the distension of the stomach (Benelam, 2009). One possibility is that the control solution ( $2 \%$ maltodextrin) is more easily discriminated from 10-SUC than from 1.5-SUC. However, rats can discriminate between sucrose and maltodextrin, a hydrolyzed starch consisting mainly of polysaccharides, both behaviorally and physiologically at a variety of concentrations (Dwyer, Lydall, \& Hayward, 2011; Nissenbaum \& Sclafani, 1987; Sako et al., 1994; Sclafani \& Nissenbaum, 1987). In addition, rats trained to self-administer the non-carbohydrate MSG, which would not be expected to overlap in taste with maltodextrin, showed similar outcomes in the satiety-specific devaluation as 1.5-SUC rats. Another possibility is that the differences in volumes of the maltodextrin control versus reinforcing solution consumed could moderate responding. Although free access to the reinforcer or control solution is by definition "drinking to satiety", the volume of pre-access fluid consumed was relatively stable across the devaluation tests in the 10-SUC and 10-E groups but changed across tests in the 1.5-SUC and MSG groups. Specifically, the MSG group consumed more control versus reinforcer solution in the second and third devaluation tests and the 1.5-SUC group consumed more control solution during the third test. In contrast, the 10-E group consumed more control than ethanol solution during each test and maintained goal-directed behavior, suggesting that simple effects of volume or stomach fullness are not driving behavior. A third possibility is that changes in pre-access consumption in the 1.5-SUC and MSG groups may reflect changes in preference over time, despite the ability of these solutions to maintain operant responding in our paradigm. Preference was not explicitly tested in the present study, but previous studies found that at low concentrations rats prefer the starch polycose to sucrose 
(Sclafani \& Clyne, 1987), supporting this interpretation of the present data. A fourth potential explanation is that the low caloric content in the 1.5-SUC and the MSG solutions renders them less effective to induce specific satiety at free access, as these solutions provided significantly fewer calories than the other reinforcer fluids. More specifically, it is possible that the taste of the solutions is sufficient to induce satiety-specific devaluation in the initial test, but subsequent tests require substantial caloric load as well. Note that in the present study we did not include a measure to confirm that the consumption of the sated reinforcer was decreased, such as providing free or operant access to the reinforcer after the 1-h exposure period. Thus, the potential contribution of post-ingestive effects of reinforcers on devaluation testing can be addressed in future studies, and may include giving pre-access to various solutions via a sham-feeding paradigm (Sclafani \& Ackroff, 2004) to separate caloric load from taste and consumption.

We found that rats trained to self-administer 10-E under the present operant conditions were goal-directed for the entire 12 weeks of testing. In contrast, Corbit, Nie, and Janak (2012) found that alcohol self-administration, as compared to $2 \%$ sucrose, promoted habit formation as measured with satiety-specific devaluation. A major difference between our work and that study is the amount of alcohol exposure: in the Corbit, Nie, and Janak study, rats had several weeks' access to alcohol in the home cage in addition to any operant access. Other studies in which alcohol exposure only occurred during operant sessions also found faster habit formation for alcohol-operant responding versus a non-drug control (Dickinson et al., 2002; Mangieri et al., 2012), although Samson and colleagues (2004) did not. Note that the aforementioned studies used variable reinforcement schedules that are either habitpromoting or known to develop habit with overtraining. However, the present study employed a fixed ratio reinforcement schedule with high perceived contingency and alternated the active lever daily. We speculate that this combination is habit-resistant, as we have yet to consistently measure habit formation with contingency degradation on this schedule (the present study; Fanelli et al., 2013; Hay et al., 2013). However, to our knowledge, direct tests of the effect of lever alternation on habit formation have not been performed.

A secondary aim of the present study was to evaluate the utility of MSG as a non-sweet reinforcer control to 10-E, as well as a potential fading solution. A common method to induce ethanol self-administration is sucrose-fading (Samson, 1986), and while this has face validity with alcohol drinking in humans, many rats stop responding when the sucrose is finally removed. While recent studies have employed methods that do not require sucrosefading (Corbit et al., 2012; Simms, Bito-Onon, Chatterjee, \& Bartlett, 2010), these methods can involve long-term home cage access or overnight operant sessions that can represent a great cost in terms of space and time. When comparing the proportion of rats meeting performance criteria, MSG was a less potent reinforcer than sucrose but equivalent to ethanol, and MSG fading was similarly effective to sucrose fading under the current conditions. The present study did not explore more subtle differences in taste memory for sucrose versus MSG that may affect later alcohol-reinforced behavior. One caveat is that the present study maintained strict performance criteria that resulted in only $30 \%$ of rats in the MSG, S-10-E, and M-10-E groups reaching criteria; higher success rates in S-10-E groups are typically found with more and longer training periods. We conclude that MSG can serve 
as a non-sweet alternate reinforcer, consistent with previous work (McCool \& Chappell, 2012), although the success rate was low under the current criteria.

In summary, these data demonstrate that studies of habit in alcohol drinking studies must carefully choose a complementary combination of non-drug rewards for control groups and tests of behavioral flexibility. For example, the present study suggests that repeated tests of the effects of satiety-specific devaluation were consistent for high-caloric solutions but not for low-caloric solutions. Thus, assessments of behavioral flexibility in alcohol selfadministration studies would benefit from multiple experimental approaches.

\section{Acknowledgments}

The authors thank Joshua Jennings, Emma Johnson, and Margot Williams for their excellent technical assistance, and thanks to Dawnya Zitzman, Rebecca Fanelli, Dr. Tatiana Shnitko, and Dr. Wendy Foulds Mathes for critical comments on the manuscript. This research was funded by a NIH ARRA supplement to R01AA018008 to D.L.R. and the UNC Bowles Center for Alcohol Studies.

\section{References}

Benelam B. Satiation, satiety and their effects on eating behaviour. Nutrition Bulletin. 2009; 34:126173.

Besheer J, Fisher KR, Lindsay TG, Cannady R. Transient increase in alcohol self-administration following a period of chronic exposure to corticosterone. Neuropharmacology. 2013; 72:139-147. [PubMed: 23643750]

Besheer J, Grondin JJ, Cannady R, Sharko AC, Faccidomo S, Hodge CW. Metabotropic glutamate receptor 5 activity in the nucleus accumbens is required for the maintenance of ethanol selfadministration in a rat genetic model of high alcohol intake. Biological Psychiatry. 2010; 67:812822. [PubMed: 19897175]

Bradfield LA, Bertran-Gonzalez J, Chieng B, Balleine BW. The thalamostriatal pathway and cholinergic control of goal-directed action: interlacing new with existing learning in the striatum. Neuron. 2013; 79:153-166. [PubMed: 23770257]

Corbit LH, Nie H, Janak PH. Habitual alcohol seeking: time course and the contribution of subregions of the dorsal striatum. Biological Psychiatry. 2012; 72:389-395. [PubMed: 22440617]

Czachowski CL, Delory MJ. Acamprosate and naltrexone treatment effects on ethanol and sucrose seeking and intake in ethanol-dependent and nondependent rats. Psychopharmacology. 2009; 204:335-348. [PubMed: 19153715]

Dickinson A. Actions and habits: the development of behavioural autonomy. Philosophical Transactions of the Royal Society of London Series B, Biological Science. 1985; 308:67-78.

Dickinson A, Wood N, Smith JW. Alcohol seeking by rats: action or habit? The Quarterly Journal of Experimental Psychology. 2002; 55:331-348. [PubMed: 12350285]

Dwyer DM, Lydall ES, Hayward AJ. Simultaneous contrast: evidence from licking microstructure and cross-solution comparisons. Journal of Experimental Psychology Animal Behavior Processes. 2011; 37:200-210. [PubMed: 21381860]

Fanelli RR, Klein JT, Reese RM, Robinson DL. Dorsomedial and dorsolateral striatum exhibit distinct phasic neuronal activity during alcohol self-administration in rats. The European Journal of Neuroscience. 2013; 38:2637-2648. [PubMed: 23763702]

Flaherty CF. Incentive contrast: A review of behavioral changes following shifts in reward. Animal Learning \& Behavior. 1982; 10:409-440.

Foy, MR.; Foy, JG. Conditioned Taste Aversion. In: Squire, LR., editor. New Encyclopedia of Neuroscience. Vol. 3. Oxford: Academic Press; 2009. p. 35-41.

Hay RA, Jennings JH, Zitzman DL, Hodge CW, Robinson DL. Specific and nonspecific effects of naltrexone on goal-directed and habitual models of alcohol seeking and drinking. Alcoholism: Clinical and Experimental Research. 2013; 37:1100-1110. 
Mangieri RA, Cofresí RU, Gonzales RA. Ethanol seeking by Long Evans rats is not always a goaldirected behavior. PLoS One. 2012; 7:e42886. [PubMed: 22870342]

McCool BA, Chappell AM. Using monosodium glutamate to initiate ethanol self-administration in inbred mouse strains. Addiction Biology. 2012; 17:121-131. [PubMed: 21054690]

Nam HW, Hinton DJ, Kang NY, Kim T, Lee MR, Oliveros A, et al. Adenosine transporter ENT1 regulates the acquisition of goal-directed behavior and ethanol drinking through $\mathrm{A} 2 \mathrm{~A}$ receptor in the dorsomedial striatum. The Journal of Neuroscience. 2013; 33:4329-4338. [PubMed: 23467349]

Nissenbaum JW, Sclafani A. Qualitative differences in polysaccharide and sugar tastes in the rat: a two-carbohydrate taste model. Neuroscience and Biobehavioral Reviews. 1987; 11:187-196. [PubMed: 3614785]

Nolan LJ, McCaughey SA, Giza BK, Rhinehart-Doty JA, Smith JC, Scott TR. Extinction of a conditioned taste aversion in rats: I. Behavioral effects. Physiology \& Behavior. 1997; 61:319323. [PubMed: 9035264]

Rolls BJ, Rolls ET, Rowe EA, Sweeney K. Sensory specific satiety in man. Physiology \& Behavior. 1981; 27:137-142. [PubMed: 7267792]

Sako N, Shimura T, Komure M, Mochizuki R, Matsuo R, Yamamoto T. Differences in taste responses to Polycose and common sugars in the rat as revealed by behavioral and electrophysiological studies. Physiology \& Behavior. 1994; 56:741-745. [PubMed: 7800742]

Samson HH. Initiation of ethanol reinforcement using a sucrose-substitution procedure in food- and water-sated rats. Alcoholism: Clinical and Experimental Research. 1986; 10:436-442.

Samson HH, Cunningham CL, Czachowski CL, Chappell A, Legg B, Shannon E. Devaluation of ethanol reinforcement. Alcohol. 2004; 32:203-212. [PubMed: 15282114]

Sclafani A, Ackroff K. The relationship between food reward and satiation revisited. Physiology \& Behavior. 2004; 82:89-95. [PubMed: 15234596]

Sclafani A, Clyne AE. Hedonic response of rats to polysaccharide and sugar solutions. Neuroscience and Biobehavioral Reviews. 1987; 11:173-180. [PubMed: 3614783]

Sclafani A, Nissenbaum JW. Taste preference thresholds for Polycose, maltose, and sucrose in rats. Neuroscience and Biobehavioral Reviews. 1987; 11:181-185. [PubMed: 3614784]

Shibata R, Kameishi M, Kondoh T, Torii K. Bilateral dopaminergic lesions in the ventral tegmental area of rats influence sucrose intake, but not umami and amino acid intake. Physiology \& Behavior. 2009; 96:667-674. [PubMed: 19174174]

Simms JA, Bito-Onon JJ, Chatterjee S, Bartlett SE. Long-Evans rats acquire operant selfadministration of $20 \%$ ethanol without sucrose fading. Neuropsychopharmacology. 2010; 35:1453-1463. [PubMed: 20200505]

Yin HH. From actions to habits: neuroadaptations leading to dependence. Alcohol Research \& Health. 2008; 31:340-347. [PubMed: 23584008]

Yin HH, Knowlton BJ. The role of the basal ganglia in habit formation. Nature Reviews Neuroscience. 2006; 7:464-476.

Yin $\mathrm{HH}$, Knowlton BJ, Balleine BW. Inactivation of dorsolateral striatum enhances sensitivity to changes in the action-outcome contingency in instrumental conditioning. Behavioural Brain Research. 2006; 166:189-196. [PubMed: 16153716] 

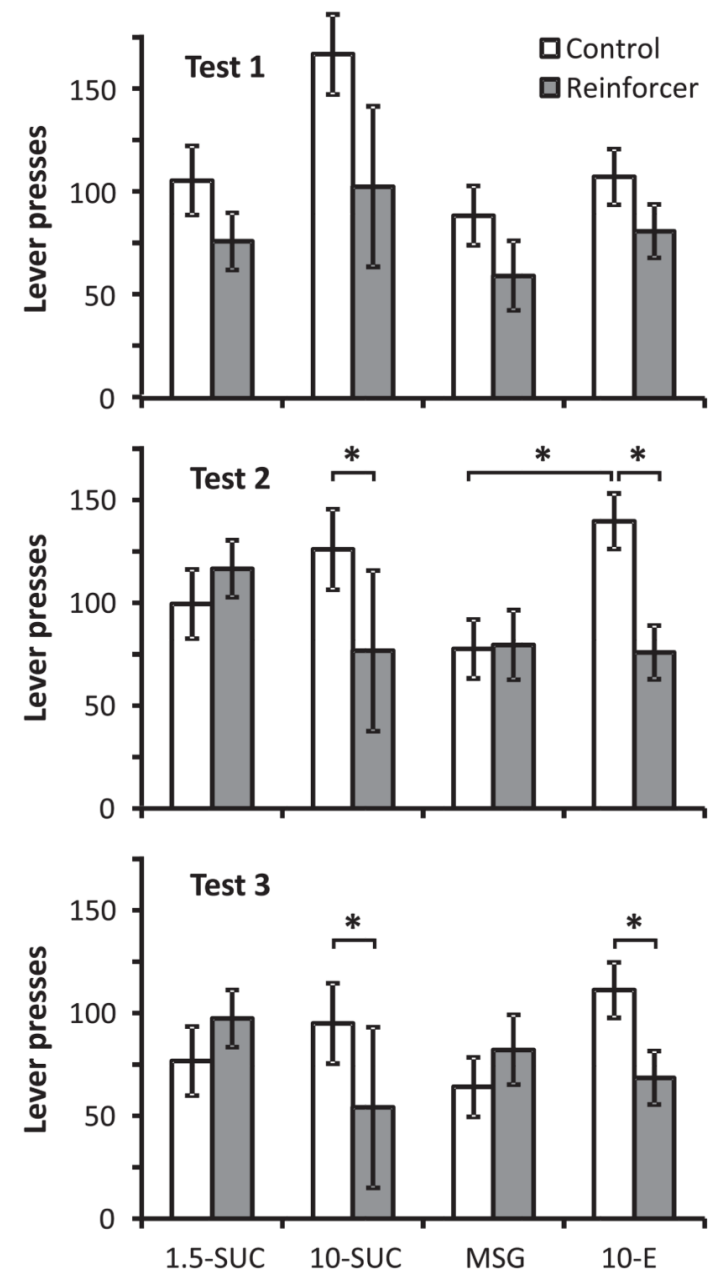

Figure 1.

Behavioral flexibility assessed by consecutive satiety-specific devaluation tests in rats trained to self-administer 1.5-SUC, 10-SUC, MSG, or 10-E. Rats received 60-min free access to the reinforcer or control solution followed by a 5-min operant session under extinction conditions. Top: Lever-press responses in extinction sessions of the first devaluation test; main effect of pre-access fluid, $p<0.001$. Middle: Responses in the extinction sessions of the second test; interaction of pre-access fluid by group, $p<0.01$. Bottom: Responses in the extinction sessions of the third test; interaction of pre-access fluid by group, $p<0.01{ }^{*} p<0.05$, Bonferroni-corrected post hoc comparison. Data presented as mean \pm S.E.M. 

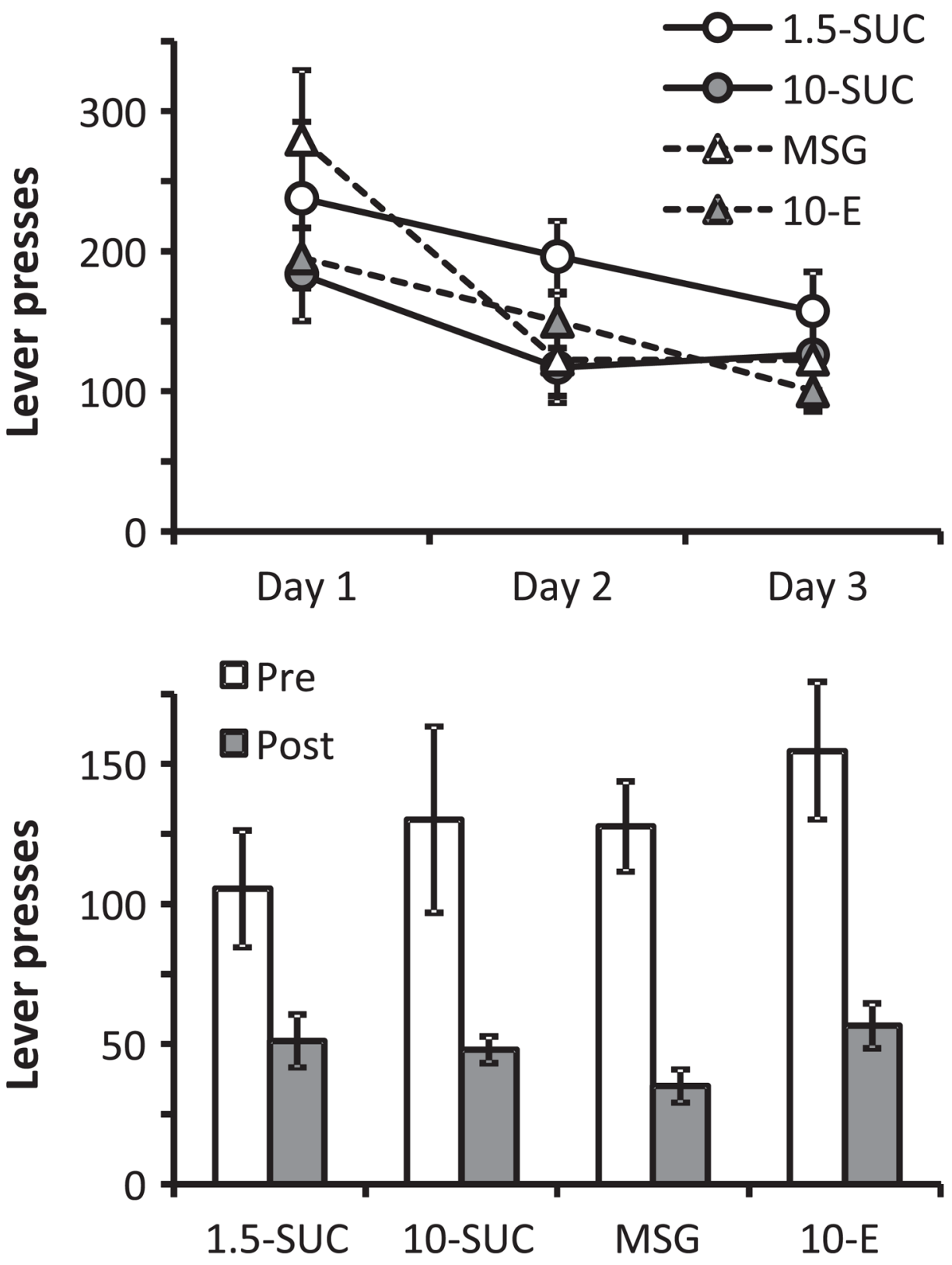

Figure 2.

Behavioral flexibility assessed by contingency-degradation training in rats trained to selfadminister 1.5-SUC, 10-SUC, MSG, or 10-E. Lever-press responding (top) during 3 days of contingency-degradation training and (bottom) in pre- and post-training extinction tests. All groups reduced responding across the 3 days of training (main effect of day, $p<0.001$ ), as well as during the post-training extinction session (main effect of pre/post session, $p<$ 0.001). Data presented as mean \pm S.E.M. 

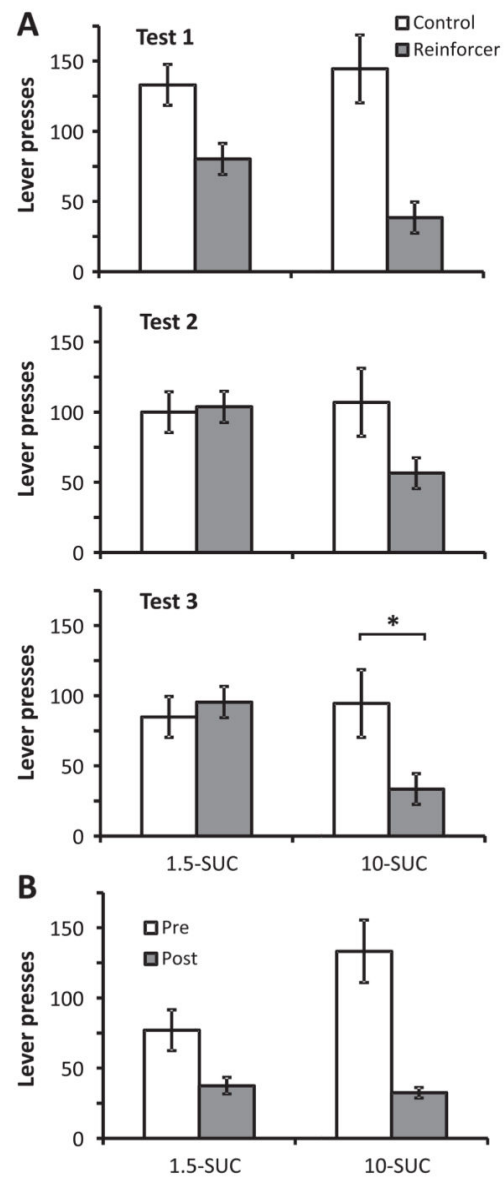

Figure 3.

Behavioral flexibility assessed by the satiety-specific devaluation test (A) and contingencydegradation training (B) in 1.5-SUC animals re-trained to self-administer 10-SUC. (A) Lever-press responses in extinction sessions after free access to the reinforcer (devaluation) or maltodextrin (control). In the first devaluation test (top), rats exhibited goal-directed behavior under both sucrose conditions (main effect of pre-access solution, $p<0.001$ ). In the second test (middle), there were no significant differences across sucrose conditions or pre-access solutions. In the third test (bottom), rats exhibited goal-directed behavior only under 10-SUC conditions (interaction of pre-access fluid by sucrose condition, $p<0.05$, ${ }^{*} p$ $<0.05$, Bonferroni-corrected post hoc comparison). (B) Lever-press responding in extinction tests before and after degradation training. Rats exhibited less reward seeking in the postversus the pre-training test, indicating goal-directed behavior, both when trained for 1.5SUC and 10-SUC reinforcement (main effect of session, $\mathrm{p}<0.005$ ). Data presented as mean \pm S.E.M. 


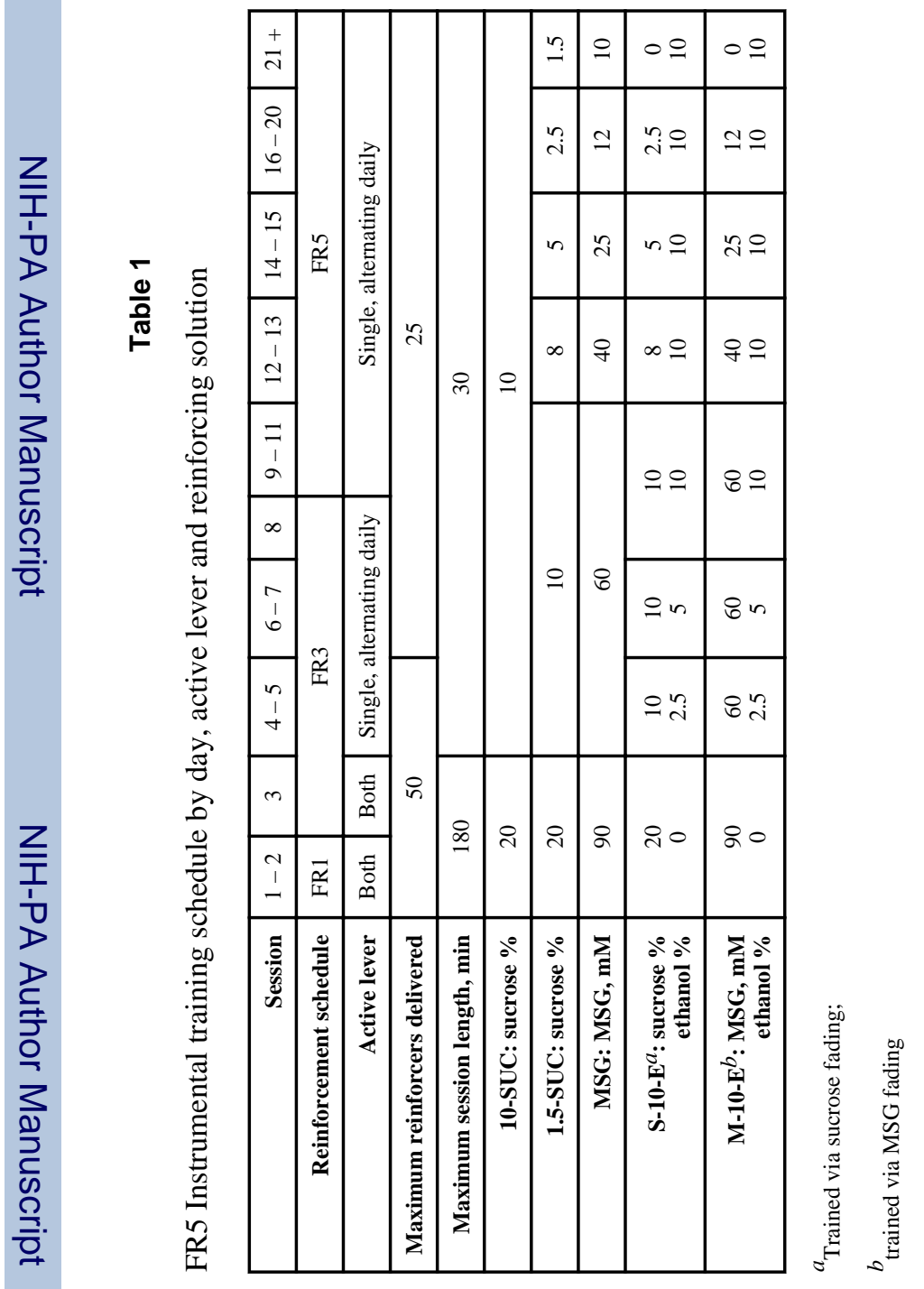




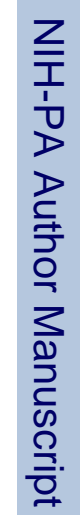

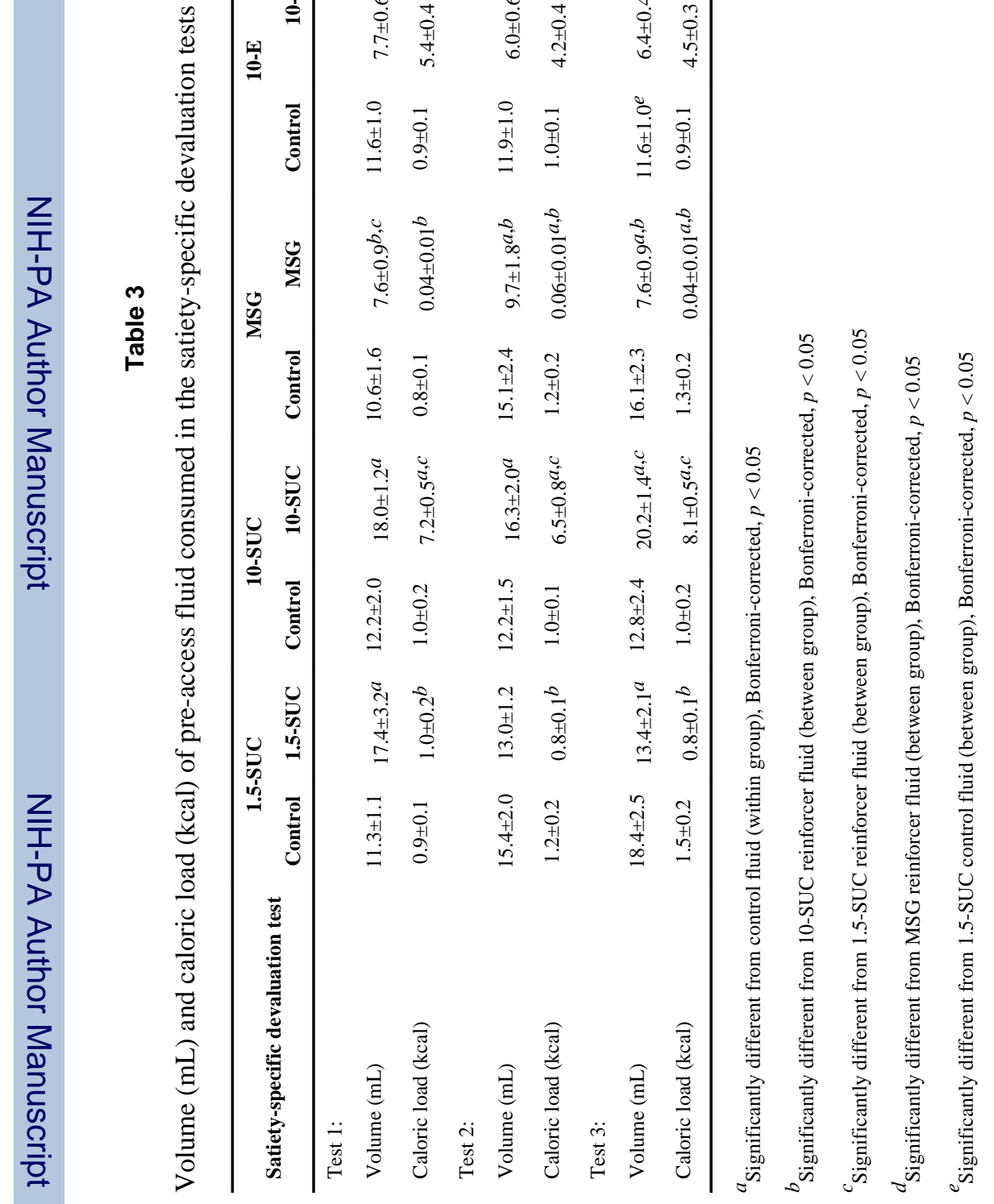

